\title{
Mechanics of instability-induced pattern transformations in elastomeric porous cylinders
}

\author{
Farhad Javid ${ }^{\mathrm{a}}$, Jia Liu ${ }^{\mathrm{a}}$, Jongmin Shim ${ }^{\mathrm{b}}$, James Weaver ${ }^{\mathrm{c}}$, Ali Shanian ${ }^{\mathrm{d}}$, \\ Katia Bertoldia,e \\ ${ }^{a}$ Harvard John A. Paulson School of Engineering and Applied Sciences, Harvard \\ University, Cambridge, MA 02138, USA \\ ${ }^{b}$ Department of Civil, Structural and Environmental Engineering, University at Buffalo, \\ Buffalo, NY 14260, USA \\ ${ }^{c}$ Wyss Institute for Biologically Inspired Engineering, Harvard University, Cambridge, \\ MA 02138, USA \\ ${ }^{d}$ Rolls-Royce Energy, Dorval, Quebec, H9P1A5, Canada \\ ${ }^{e}$ Kavli Institute, Harvard University, Cambridge, MA 02138, USA
}

\begin{abstract}
In this paper, we combine experiments and numerical simulations to investigate the large deformation mechanics of periodically patterned cylindrical structures under uniaxial compression. Focusing on cylinders with a square array of circular pores, we show that their buckling behavior is not only controlled by the porosity (as for the case of the corresponding infinitely large planar structures), but also by the length and thickness of the shell and the number of pores along the full circumference. While infinitely long cylindrical shells only support long wavelength (global) modes, by reducing the length and tuning the thickness, short wavelength (local) modes can be observed. Furthermore, frustrated short wavelength modes are triggered when a local instability is critical, but the buckling pattern is not compatible with the number of pores along the circumference.
\end{abstract}

Keywords: Instability, Pattern transformation, Large deformation, 
Frustration, Porous structure, Cylindrical shell

\section{Introduction}

Structural materials are often inhomogeneous on small scales and possess specific microstructures. In particular, porous materials with well-defined periodicity are ubiquitous not only in nature, but also in synthetic structures and devices (Gibson and Ashby, 1999). Periodic porous materials are used to design lightweight structures (Queheillalt and Wadley, 2005) with exceptional mechanical characteristics such as high energy absorption (Wierzbicki and Abramowicz, 1983; Papka and Kyriakides, 1994) and excellent acoustic damping (Verdejo et al., 2009). Moreover, these materials are attractive media for controlling and manipulating the propagation of waves with applications ranging from optical fibers and sound filters to photonic integrated circuits and acoustic mirrors (Maldovan and Thomas, 2009).

Periodic porous structures made of elastic materials are capable of undertaking homogeneous and reversible pattern transformations under compression due to the buckling of their beam-like ligaments. For example, upon reaching a critical applied deformation a square array of circular holes in a 2D elastomeric matrix suddenly transforms into a periodic pattern of alternating, mutually orthogonal ellipses (Mullin et al., 2007; Michel et al., 2007; Zhang et al., 2008). Such pattern transformation has been found to be robust and only marginally affected by small imperfections and edge effects (Bertoldi et al., 2008). However, its emergence can be compromised in structures characterized either by low levels of porosity or by multiple nucleation sites (Bertoldi et al., 2010; Zhu, 2011; Kang et al., 2013). In fact, it has been 
found that by progressively reducing the porosity a transition from instabilities with a short wavelength to instabilities characterized by a wavelength much larger than the scale of the microstructure occurs (Bertoldi et al., 2010). Moreover, the presence of multiple nucleation sites has been shown to result in domains of uniform buckling patterns separated by antiphase boundaries (Zhu, 2011; Kang et al., 2013).

Motivated by recent studies on porous shells that derive functionality from mechanical instabilities (Shim et al., 2012; Lazarus and Reis, 2015), here we investigate the non-linear response under uniaxial compression of elastomeric cylindrical structures patterned with a square array of circular pores. Our combined experimental and numerical results show three key features. First, differently from the case of an infinite 2D matrix perforated with a square array of circular pores that supports short wavelength modes for large enough values of porosity (Michel et al., 2007; Bertoldi et al., 2008), only long wavelength modes can be triggered in infinitely long patterned cylinders. Second, by reducing the length of the cylindrical shells and tuning its thickness and porosity, short wavelength modes resulting in a checkerboard pattern similar to that found in their 2D counterparts can be observed. Third, the response of the cylindrical shells is also significantly affected by the number of pores along the full circumference. More specifically, frustrated short wavelength modes characterized by a line of defects can be observed in samples with an odd number of pores along the circumference. In contrast to the 2D periodic structures, the line of defects is not introduced because of multiple nucleation sites, but it is due to the incompatibility between the periodicity of the short wavelength mode and the odd number of 
pores along the circumference.

This paper is organized as follows. After presenting the family of porous cylindrical structures considered in this study (Section 2), in Section 3 we describe the experiments conducted by subjecting three different elastomeric structures to uniaxial compression. Then, in Section 4 we explain the numerical analyses that are used to investigate the nonlinear response of the structures on both full size and unit cell models. Finally, numerical and experimental results are compared and discussed in Section 5, highlighting the effect of the cylinder length, thickness, porosity and number of pores along the full circumference on the response of the structure.

\section{Geometry}

A schematic of the porous cylindrical structures considered in this study is shown in Fig. 1a. The cylinder has an inner radius $R_{\mathrm{i}}$, an outer radius $R_{\mathrm{o}}$ and a square array of circular pores around the full circumference. The structure is, therefore, characterized by the unit cell shown in Fig. 1b, consisting of a cylindrical sector with a single pore. If the number of pores around the full circumference of the cylinder is denoted by $N_{\mathrm{c}}$, the angle of this sector is defined as

$$
\alpha=2 \pi / N_{\mathrm{c}}
$$

and its length, $L_{\mathrm{u}}$, is given by

$$
L_{\mathrm{u}}=\alpha R_{\mathrm{o}}
$$

Moreover, the size of the pores is defined by the angle $\alpha_{\mathrm{p}}$ (see Fig. 1b). 
a)

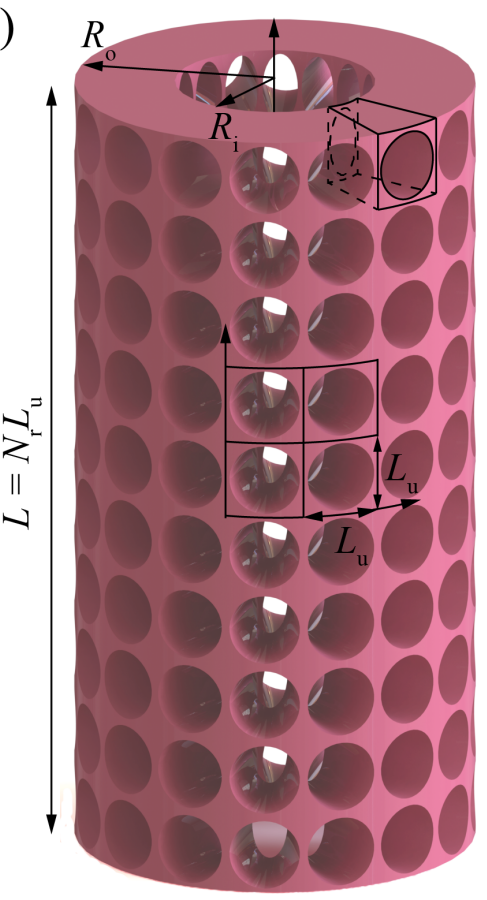

b)
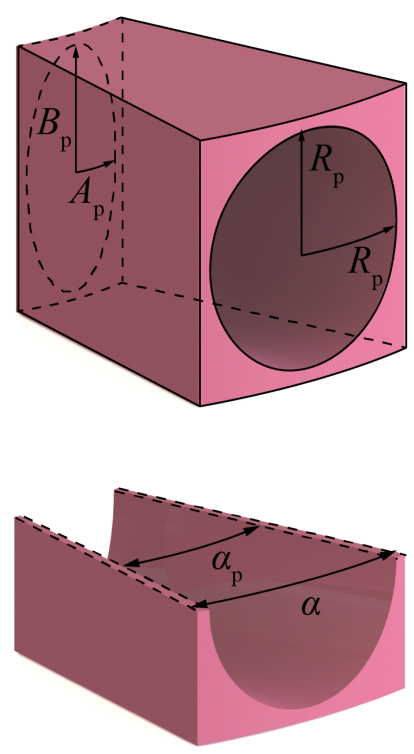

Figure 1: Periodic porous cylinder with a square of circular pores: (a) schematic of the full size structure and (b) schematic of the unit cell. 
Since the pores are characterized by a fixed value of $\alpha_{\mathrm{p}}$, their cross-section changes from circular on the outer surface to elliptical on the inner one. More specifically, if we denote with $R_{\mathrm{p}}=\alpha_{\mathrm{p}} R_{\mathrm{o}} / 2$ the radius of the circular pores on the outer surface, the semi-axes of the elliptical voids on the inner surface are given by

$$
A_{\mathrm{p}}\left(R_{\mathrm{i}}\right)=\frac{\alpha_{\mathrm{p}}}{2} R_{\mathrm{i}}, \quad \text { and } \quad B_{\mathrm{p}}\left(R_{\mathrm{i}}\right)=R_{\mathrm{p}}=\frac{\alpha_{\mathrm{p}}}{2} R_{\mathrm{o}} .
$$

Finally, the porosity of the structure can be obtained as

$$
\psi=\frac{\int_{R_{i}}^{R_{o}} \pi A_{p}(R) B_{p}(R) d R}{L_{u} \frac{\alpha}{2}\left(R_{o}^{2}-R_{i}^{2}\right)}=\frac{\int_{R_{i}}^{R_{o}} \pi \frac{\alpha_{p}}{2} R \frac{\alpha_{p}}{2} R_{o} d R}{\alpha R_{o} \frac{\alpha}{2}\left(R_{o}^{2}-R_{i}^{2}\right)}=\frac{\pi \alpha_{p}^{2}}{4 \alpha^{2}} .
$$

\section{Experiments}

Three specimens with different microstructures are fabricated using a molding approach. Each specimen is made of $N_{\mathrm{r}}=10$ rows of pores and is characterized by $R_{\mathrm{i}}=12.5 \mathrm{~mm}$ and $R_{\mathrm{o}}=25 \mathrm{~mm}$. The other geometrical parameters defining the three samples are provided in Table 1, where $L=$ $N_{\mathrm{r}} L_{u}$ denotes the total length of the specimens.

Table 1: Design parameters of casted specimens.

\begin{tabular}{lcccc}
\hline & $N_{\mathrm{c}}$ & $\alpha_{\mathrm{p}}(\mathrm{rad})$ & $\psi$ & $L(\mathrm{~mm})$ \\
\hline Specimen 1 & 16 & 0.353 & 0.636 & 98.17 \\
Specimen 2 & 15 & 0.377 & 0.636 & 104.72 \\
Specimen 3 & 18 & 0.271 & 0.471 & 87.27 \\
\hline
\end{tabular}

To manufacture the samples, we start by fabricating a negative mold 
using a 3D printer (Connex 500 available from Objet, Ltd.) with VeroBlue (product number: RGD840, Objet) material. Note that the mold of specimen 2 is designed to cast one third of the cylinder while the other two are designed to cast half cylinders. The specimens are cast using a silicone rubber material (Elite Double, SHORE-A 8; Zhermack). Before replication, a releasing agent (Easy Release 200 available from Smooth-On, Inc.) is sprayed on the mold surfaces for easy separation. The cast mixture is first placed in vacuum for degassing for around five minutes and, then, set at room temperature for curing. After demolding, each cylindrical structure is built by joining all its parts with a silicone adhesive sealant (DAP Products Inc., Baltimore, MD).

Each specimen is tested under uniaxial compression using a Discovery Hybrid rheometer (TA Instruments, New Castle, DE). All tests are conducted at $0.1 \mathrm{~mm} \cdot \mathrm{s}^{-1}$, ensuring quasi-static conditions. Before performing the tests, the samples are glued to flat plate fixtures using an instant glue (Krazy Glue, Westerville, $\mathrm{OH}$ ) to prevent slippage at their ends. During each test, the applied load versus deformation is recorded and then compared to the numerical results obtained by conducting the analysis described in Section 4 .

Differently from the case of uniaxial tension and pure torsion for which all samples deform similarly (see Appendix A), the experiments indicate that under uniaxial compression the microstructure has a profound effect and significantly alters the deformation path followed by the cylinders. In fact, as shown in Fig. 2, three different post-buckling behaviors are found under compression. In specimen 3, characterized by a low value of porosity $(\psi \sim 0.47)$, a global buckling mode with a wavelength equal to the length of the sample is observed. By contrast, in specimens 1 and 2, characterized 
by a higher value of porosity $(\psi \sim 0.6)$, buckling causes a sudden transformation in the periodic pattern of the structure, without altering the cylinder global shape. More specifically, in specimen 1 we observe the formation of a checkerboard pattern identical to the one previously observed in $2 \mathrm{D}$ periodic structures comprising a square array of circular pores (Mullin et al., 2007; Bertoldi et al., 2008; Zhang et al., 2008) and characterized by a wavelength twice the length of the unit cell in both circumferential and axial directions (as highlighted by the yellow line on specimen 1 in Fig. 2). However, in specimen 2 the formation of such pattern is constrained by the odd number of pores around the full circumference $\left(N_{\mathrm{c}}=15\right)$. As a result, a line of defects appears in the structure, as highlighted by the yellow contour lines on specimen 2 in Fig. 2. It should be noted that similar defect lines induced by buckling have also been observed in 2D periodic structures (Kang et al., 2013; Zhu, 2011). However, while in 2D structures these are induced by buckling patterns evolving from different nucleation sites, here they are the result of the incompatibility between the periodicity of the structure and the checkerboard pattern.

\section{Modeling}

To study the large deformation mechanics of cylindrical porous structures under compression, nonlinear simulations are performed with the finite element package, ABAQUS/Standard using both full size and unit cell models. All models are constructed using 10-node modified tetrahedral elements with hourglass control (C3D10M element type). The accuracy of the mesh is insured by a mesh refinement study, resulting in $\sim 900$ elements for each unit 

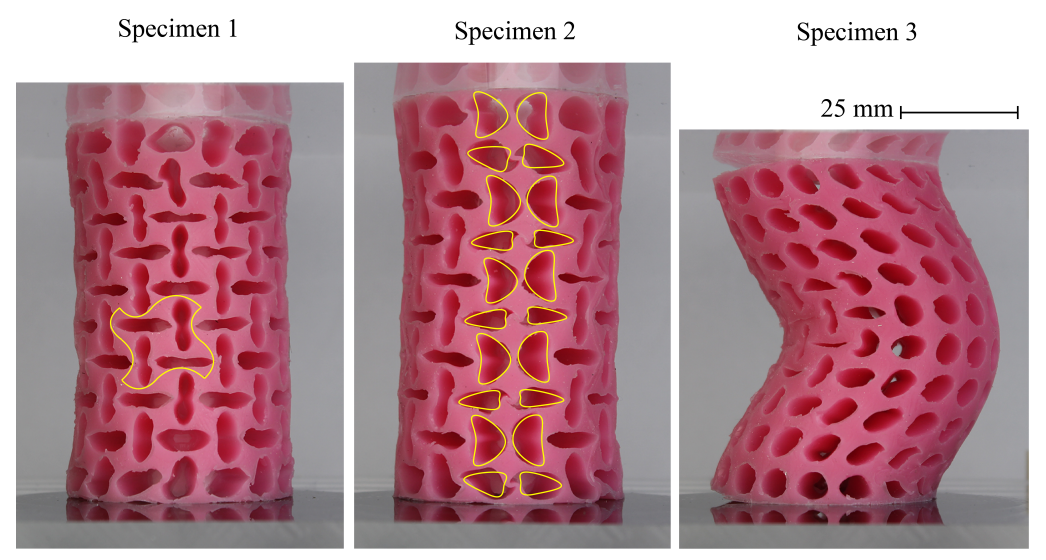

Figure 2: Buckling pattern of the specimens under high compressive strains $(\varepsilon=0.15$ for all samples): specimen 1 shows a local buckling pattern, specimen 2 shows a local buckling pattern with a row of frustrated pores; and specimen 3 shows a global buckling pattern.

cell (note that the full size models of the three specimens are constructed by patterning the corresponding unit cell in both axial and circumferential directions). In all simulations the response of the silicon rubber used to fabricated the samples is captured using an almost incompressible hyperelastic neo-Hookean model with initial shear modulus $\mu=82.5 \mathrm{kPa}$ and bulk modulus $K=41.25 \mathrm{MPa}$.

\subsection{Boundary conditions}

Here, we first present the boundary conditions used to investigate the response of the full size models and then focus on the periodic boundary conditions used for the unit cell analysis.

\subsubsection{Full size model}

To simulate the experimental conditions in the numerical analysis and mimic the glue attachment of the samples to the loading fixtures, we completely fix the nodes on the bottom surfaces of the cylinders. Moreover, the 
models are subjected to uniaxial compression by fixing all nodes on their top surfaces in radial and circumferential direction, while uniformly displacing them in axial direction. The applied nominal strain, $\varepsilon$, is then obtained as the ratio between the applied axial displacement and the initial length of the model, $L$. Moreover, the corresponding nominal stress, $S$, is calculated by dividing the total reaction force on the top surface by the undeformed cross-sectional area of the sample, $\pi\left(R_{o}^{2}-R_{i}^{2}\right)$.

\subsubsection{Unit cell}

To reduce the computational cost, we take advantage of the periodicity of the structures and investigate their response using the unit cell shown in Fig. 1b with periodic boundary conditions defined on four of its surfaces. In fact, the structure is periodic along the circumferential and axial directions, while it is not in the radial direction.

To define suitable boundary conditions, we introduce a cylindrical coordinate system, fixed to the reference frame, and denote with $\mathbf{Y}=(R, \Theta, Z)$ and $\mathbf{y}=(r, \theta, z)$ the coordinates of a material point in the undeformed (reference) and deformed configuration, respectively. The change in coordinates of an arbitrary point is then given by,

$$
\mathbf{D}=\left[D_{R}, D_{\Theta}, D_{Z}\right]=\mathbf{y}-\mathbf{Y}=[(r-R),(\theta-\Theta),(z-Z)] .
$$

Moreover, an infinitesimal change in coordinates induced by the applied deformation can be expressed as

$$
\mathrm{d} \mathbf{D}=\mathbf{J} \mathrm{d} \mathbf{Y}
$$


where

$$
\mathbf{J}=\left[\begin{array}{ccc}
J_{11} & J_{12} & J_{13} \\
J_{21} & J_{22} & J_{23} \\
J_{31} & J_{32} & J_{33}
\end{array}\right]=\left[\begin{array}{ccc}
\frac{\partial D_{R}}{\partial R} & \frac{\partial D_{R}}{\partial \Theta} & \frac{\partial D_{R}}{\partial Z} \\
\frac{\partial D_{\Theta}}{\partial R} & \frac{\partial D_{\Theta}}{\partial \Theta} & \frac{\partial D_{\Theta}}{\partial Z} \\
\frac{\partial D_{Z}}{\partial R} & \frac{\partial D_{Z}}{\partial \Theta} & \frac{\partial D_{Z}}{\partial Z}
\end{array}\right]
$$

Next, we consider a macroscopic deformation represented by $\overline{\mathbf{J}}$ applied to the unit cell and focus on pairs of nodes periodically located on the top/bottom and left/right surfaces. Making use of Eq. (6), the coordinates of two nodes periodically located on the top and bottom faces can be related as

$$
\begin{aligned}
D_{R}^{t}-D_{R}^{b} & =\bar{J}_{13}\left[Z^{t}-Z^{b}\right], \\
D_{\Theta}^{t}-D_{\Theta}^{b} & =\bar{J}_{23}\left[Z^{t}-Z^{b}\right], \\
D_{Z}^{t}-D_{Z}^{b} & =\bar{J}_{33}\left[Z^{t}-Z^{b}\right],
\end{aligned}
$$

since $R^{t}=R^{b}$ and $\Theta^{t}=\Theta^{b}$. Note that the superscripts $t$ and $b$ denote quantities associated to nodes on the top and bottom faces, respectively.

Moreover, since the cylindrical structure is rotationally periodic and consists of $N_{\mathrm{c}}$ identical unit cells forming a closed ring, it is easy to show that for two nodes periodically located on the left and right faces,

$$
\mathbf{D}^{r}-\mathbf{D}^{l}=\mathbf{0} .
$$

Here, the superscripts $r$ and $l$ refer to quantities associated to nodes on the right and left surfaces, respectively. In fact, if we assume that for the $\alpha$-th unit cell along the circumference $\mathbf{D}^{l, \alpha}-\mathbf{D}^{r, \alpha}=\mathbf{Q}(\mathbf{Q}$ being an arbitrary 
vector $)$, then $\sum_{\alpha=1}^{N_{\mathrm{c}}}\left(\mathbf{D}^{l, \alpha}-\mathbf{D}^{r, \alpha}\right)=N_{\mathrm{c}} \mathbf{Q}$. However, since $\mathbf{D}^{l, \alpha}=\mathbf{D}^{r, \alpha-1}$ and $\mathbf{D}^{r, \alpha}=\mathbf{D}^{l, \alpha+1}$, it follows that $\sum_{\alpha=1}^{N_{\mathrm{c}}}\left(\mathbf{D}^{l, \alpha}-\mathbf{D}^{r, \alpha}\right)=\mathbf{0}$, showing the only possibility is $\mathbf{Q}=\mathbf{0}$.

Operationally, we use a user defined multiple point constraint (MPC) subroutine to implement Eqs. (8) and (9) in our numerical simulations. More specifically, in the MPC subroutine Eqs. (8) and (9) are used to define relations between the displacements of pairs of nodes periodically located on the top/bottom and left/right surfaces. In fact, since for a generic node

$$
\begin{gathered}
u_{R}=\left(R+D_{R}\right) \cos \left(D_{\Theta}\right)-R, \\
u_{\Theta}=\left(R+D_{R}\right) \sin \left(D_{\Theta}\right), \\
u_{Z}=D_{Z},
\end{gathered}
$$

Eqs. (8) can be rewritten as

$$
\begin{gathered}
\sqrt{\left[R^{t}+u_{R}^{t}\right]^{2}+\left(u_{\Theta}^{t}\right)^{2}}-\sqrt{\left[R^{b}+u_{R}^{b}\right]^{2}+\left(u_{\Theta}^{b}\right)^{2}}=\bar{J}_{13}\left[Z^{t}-Z^{b}\right] \\
\arctan \left(\frac{u_{\Theta}^{t}}{R^{t}+u_{R}^{t}}\right)-\arctan \left(\frac{u_{\Theta}^{b}}{R^{b}+u_{R}^{b}}\right)=\bar{J}_{23}\left[Z^{t}-Z^{b}\right] \\
u_{Z}^{t}-u_{Z}^{b}=\bar{J}_{33}\left[Z^{t}-Z^{b}\right] .
\end{gathered}
$$

and Eqs. (9) reduce to

$$
\begin{aligned}
& u_{R}^{l}-u_{R}^{r}=0, \\
& u_{\Theta}^{l}-u_{\Theta}^{r}=0, \\
& u_{Z}^{l}-u_{Z}^{r}=0 .
\end{aligned}
$$


Moreover, we define a virtual node and use its degrees of freedom to prescribe $\left(\bar{J}_{13}, \bar{J}_{23}, \bar{J}_{33}\right)$.

To simulate uniaxial compression, we prescribe $\left(\bar{J}_{13}, \bar{J}_{23}, \bar{J}_{33}\right)=(0,0, \varepsilon)$ and extract the corresponding macroscopic nominal stress, $\bar{S}$, through virtual work considerations. We start by calculating the external and internal virtual work as

$$
\delta W_{e x t}=\xi_{3} \delta \bar{J}_{33},
$$

and

$$
\delta W_{i n t}=V_{0} \bar{S} \delta \bar{F}_{33}=V_{0} \bar{S} \delta \bar{J}_{33},
$$

where $\xi_{3}$ is the reaction force at the virtual node corresponding to the as-

signed displacement $\bar{J}_{33}$ and $V_{0}$ denotes the volume of the unit cell in the undeformed configuration. Since $\delta W_{\text {ext }}=\delta W_{\text {int }}$, it follows that

$$
\bar{S}=\frac{\xi_{3}}{V_{0}} .
$$

Note that the periodic boundary conditions derived above are validated by comparison with analytical expressions for the applied force versus deformation obtained for a homogeneous (non-porous) hollow cylinder subjected to uniaxial loading and pure torsion (see Appendix B).

\subsection{Instability analysis}

Here, we describe the instability analyses that we perform on the full size models and their corresponding unit cells. 


\subsubsection{Full size models}

The stability of the full size models is examined using eigenvalue analyses. More specifically, a linear perturbation procedure is accomplished in ABAQUS/Standard using the *BUCKLE module.

\subsubsection{Unit cell models}

Although buckling often alters the periodicity of the structure, it may be still investigated considering an initial unit cell and studying the propagation of small-amplitude perturbations of arbitrary wavelength superimposed on the current state of deformation (Williams and Anderson, 1983; Triantafyllidis et al., 2006; Bertoldi et al., 2008; Ning and Pellegrino, 2015). While a real natural frequency corresponds to a propagating wave, a complex natural frequency identifies a perturbation exponentially growing with time. Therefore, the transition between a stable and an unstable configuration is identified when the frequency vanishes.

To conduct such analysis, we first uniaxially compress the unit cell by applying a finite strain, $\varepsilon$, and then investigate the propagation of small amplitude elastic waves in the predeformed structure. Since the structure is periodic along the circumferential and axial directions, the buckling mode follows the Bloch wave relation (Kittel, 2005),

$$
\mathbf{u}\left(R, \Theta+\alpha, Z+L_{u}\right)=\mathbf{u}(R, \Theta, Z) \exp \left(i k_{\Theta} \alpha+i k_{Z} L_{u}\right)
$$

where $\mathbf{u}=\left(u_{R}, u_{\Theta}, u_{Z}\right), k_{\Theta}=2 \pi /\left(\alpha m_{\Theta}\right)=N_{c} / m_{\Theta}, k_{Z}=2 \pi /\left(L_{u} m_{Z}\right)$. Moreover, $m_{\Theta}$ and $m_{Z}$ are the number of unit cells contained in a full wavelength along the circumferential and axial directions, respectively. It follows from 
Eq. (16) that $^{1}$

$$
\mathbf{u}^{t}=\mathbf{u}^{b} \exp \left(i \frac{2 \pi}{m_{Z}}\right), \quad \mathbf{u}^{r}=\mathbf{u}^{l} \exp \left(i \frac{2 \pi}{m_{\Theta}}\right)
$$

and an instability is detected at the lowest value of $\varepsilon$ for which $m_{Z}$ and $m_{\Theta}$ exist such that the corresponding wave has vanishing frequency.

It is important to note that the length of the cylinder limits the possible values of $m_{Z}$ to be considered in the analysis. In fact, although larger values of $m_{Z}$ result in lower critical strains (since longer cylinders are easier to buckle), only buckling modes with a wavelength shorter of or equal to the length of the structure can be observed in the porous cylinders. Therefore, we consider $1 \leq m_{Z} \leq N_{\mathrm{r}}$. Moreover, since the cylindrical structure consists of $N_{\mathrm{c}}$ unit cells forming a closed ring (Prasad et al., 1974; Thomas, 1979; Williams and Anderson, 1983; Ning and Pellegrino, 2015)

$$
\mathbf{u}\left(R, \Theta+N_{c} \alpha, Z\right)=\mathbf{u}(R, \Theta, Z)
$$

Making use of Eq. (16), it is easy to show that Eq. (18) is satisfied only if

$$
\exp \left(i k_{\Theta} N_{c} \alpha\right)=\exp \left(i \frac{2 \pi}{m_{\Theta}} N_{c}\right)=1
$$

yielding

$$
m_{\Theta}=\frac{N_{c}}{n},
$$

where $n$ is an integer number. Furthermore, since $k_{\Theta} \alpha=2 \pi / m_{\Theta}=2 \pi n / N_{C} \in$

\footnotetext{
${ }^{1}$ Note that Eqs. (17) are equivalent to $\mathbf{D}^{t}=\mathbf{D}^{b} \exp \left(i 2 \pi / m_{Z}\right)$, and $\mathbf{D}^{r}=$ $\mathbf{D}^{l} \exp \left(i 2 \pi / m_{\Theta}\right)$, since linearization of Eqs. (10) yields $u_{R} \sim D_{R}$ and $u_{\Theta} \sim R D_{\Theta}$.
} 
$\left[0,2 \pi\left[\right.\right.$, it follows that there are $N_{c}$ values of $n$ to be considered (i.e. $n=$ $\left.0,1,2, \ldots, N_{c}-1\right)$. However, since $\exp \left(i 2 \pi n / N_{c}\right)=\exp \left(-i 2 \pi\left(N_{c}-n\right) / N_{c}\right)$, $n$ and $N_{c}-n$ are associated with waves of the same form (but with opposite direction of propagation). Therefore, we only need to consider

$$
n=0,1,2, \ldots . .\left\lfloor N_{\mathrm{c}} / 2\right\rfloor
$$

where $\left\lfloor N_{\mathrm{c}} / 2\right\rfloor$ denotes the largest integer smaller than or equal $N_{\mathrm{c}} / 2$.

Finally, to work with the complex-valued relations of the Bloch-periodic conditions (17) in a commercial software such as ABAQUS/Standard, we split all fields into real and imaginary parts (Aberg and Gudmundson, 1997; Bertoldi et al., 2008). These two meshes are, then, coupled by Bloch-periodic displacement boundary conditions:

$$
\begin{aligned}
\operatorname{Real}\left(\mathbf{u}^{t}\right) & =\operatorname{Real}\left(\mathbf{u}^{b}\right) \cos \left[\frac{2 \pi}{m_{Z}}\right]-\operatorname{Imag}\left(\mathbf{u}^{b}\right) \sin \left[\frac{2 \pi}{m_{Z}}\right], \\
\operatorname{Imag}\left(\mathbf{u}^{t}\right) & =\operatorname{Real}\left(\mathbf{u}^{b}\right) \sin \left[\frac{2 \pi}{m_{Z}}\right]+\operatorname{Imag}\left(\mathbf{u}^{b}\right) \cos \left[\frac{2 \pi}{m_{Z}}\right], \\
\operatorname{Real}\left(\mathbf{u}^{r}\right) & =\operatorname{Real}\left(\mathbf{u}^{l}\right) \cos \left[\frac{2 \pi}{m_{\Theta}}\right]-\operatorname{Imag}\left(\mathbf{u}^{l}\right) \sin \left[\frac{2 \pi}{m_{\Theta}}\right], \\
\operatorname{Imag}\left(\mathbf{u}^{r}\right) & =\operatorname{Real}\left(\mathbf{u}^{l}\right) \sin \left[\frac{2 \pi}{m_{\Theta}}\right]+\operatorname{Imag}\left(\mathbf{u}^{l}\right) \cos \left[\frac{2 \pi}{m_{\Theta}}\right] .
\end{aligned}
$$

Note that Eqs. (22) can be easily implemented within ABAQUS/Standard using a MPC user-subroutine. 


\section{Results}

To investigate the response of the porous cylindrical structures under uniaxial compression, we first study their stability performing eigenvalue and Bloch wave analysis for the full size and unit cell models, respectively (as described in Section 4.2). We then conduct post-buckling analyses by introducing a geometrical imperfection with the form of the critical eigenmode to preferentially activate the first mode during simulation of the compression test.

\subsection{Instability analysis}

Full size models. The first eigenmode and corresponding critical compressive strain for all three specimens are shown in Fig. 3 and clearly correspond to the experimentally observed buckling patterns (see Fig. 2). To better characterize these numerically obtained modes, we focus on a circular path in the middle of the models (highlighted in yellow in the insets in Fig. 4d-f), plot $u_{R}, u_{\Theta}$ and $u_{Z}$ along this path for all three specimens (Figs. 4a-c), and decompose these displacements into their Fourier components (Figs. 4d-f). The amplitude spectra of the displacement components indicate that the buckling patterns of specimens 1 and 2 are characterized by $m_{\Theta}=N_{\mathrm{c}} / n=16 / 8=2$ (see Fig. 4d) and $m_{\Theta}=15 / 7^{2}$ (see Fig. 4e), respectively. As shown in Fig. 3, the buckling modes of both specimens are characterized by a local pattern transformation which alters the periodicity of the structure. However, the wavelength of these patterns along the circumference is different, since the

\footnotetext{
${ }^{2}$ Note that $n=7$ and 8 are associated with the same buckling pattern, since $\exp \left(i 2 \pi 7 / N_{c}\right)=\exp \left(-i 2 \pi\left(N_{c}-8\right) / N_{c}\right)$ for $N_{c}=15$.
} 
checkerboard pattern characterized by $m_{\Theta}=2$ that emerges in specimen 1 is not compatible with the odd number of pores of specimen 2. Finally, as in the experiments, for specimen 3 the critical mode is global (i.e. it is characterized by $m_{\Theta}=18$ - see Fig. 4f) and does not alter significantly the pore shapes, but, conversely, leads to a change in the overall shape of the cylinder.

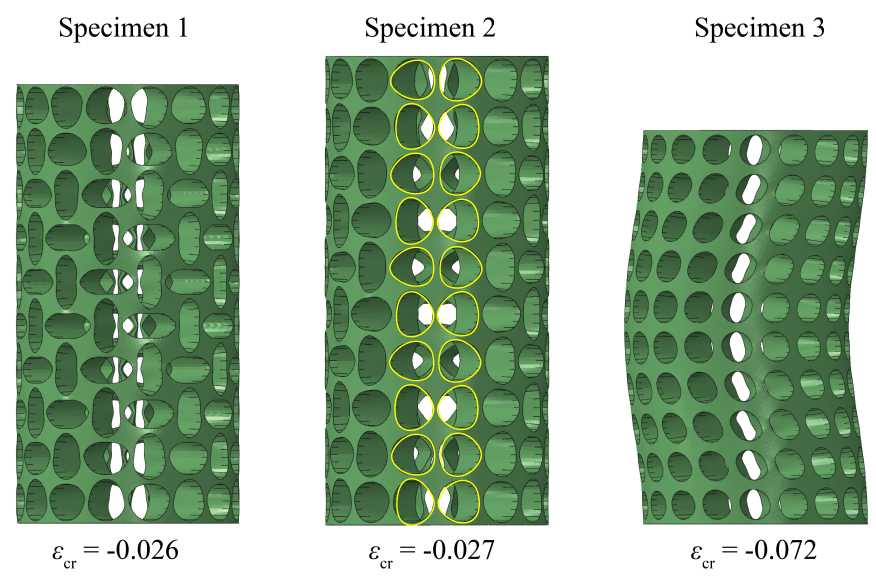

Figure 3: Eigenmodes and eigenvalues (critical strain) for the full size models.

Unit cell models. The results of the Bloch wave analyses performed on the unit cells are shown in Fig. 5 for all three specimens. In the plots, we report the evolution of the frequency $\omega$ (normalized by its maximum value, $\omega_{\max }$, which occurs at $\varepsilon=0$ ) as a function of the applied strain $\varepsilon$ for three different periodicities, $\mathbf{m}=\left(m_{\Theta}, m_{Z}\right)$, including the one which goes to zero at the lowest strain value (i.e. the critical one) $)^{3}$. In the undeformed configuration (i.e. $\varepsilon=0)$ all eigen-frequencies $\omega$ associated with the possible $\mathbf{m}$ are positive.

\footnotetext{
${ }^{3}$ Note that in our analyses we considered all possible periodicities, $\mathbf{m}$.
} 

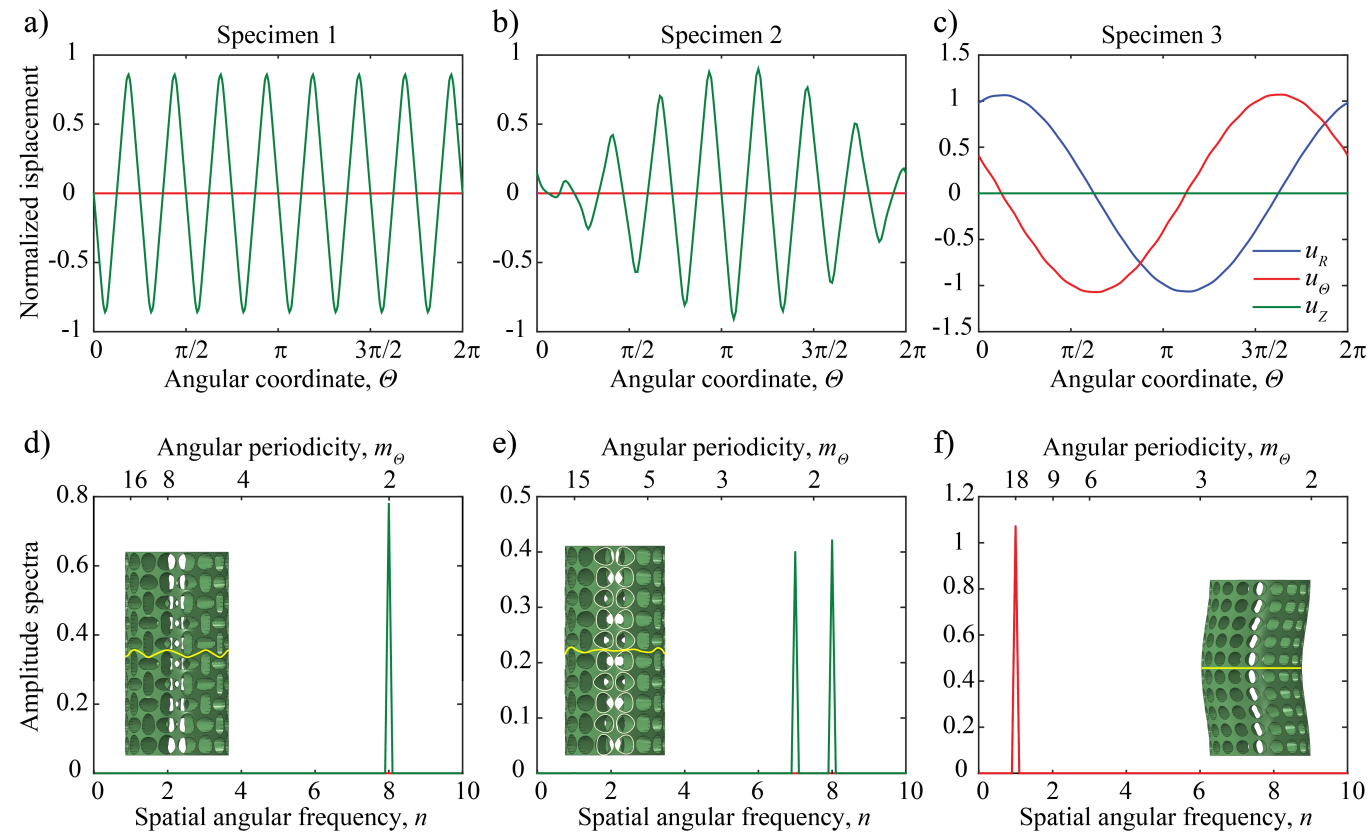

Figure 4: Frequency content of the eigenmodes for full size models. We focus on a circular path in the middle of the specimens, as highlighted by the yellow line in the models shown as insets, and report the radial, $u_{R}$, angular, $u_{\Theta}$, and axial, $u_{Z}$, displacements along this path for the three specimens (a-c). Amplitude spectra of displacements are shown in (d-f). 
However, as $\varepsilon$ increases, the eigen-frequencies associated with each $\mathbf{m}$ gradually decrease and eventually become negative. The critical strain parameter related to each periodicity, $\mathbf{m}$, can be easily extracted from the plots, since it corresponds to the intersection point between each curve and the horizontal line $\omega=0$. Finally, the onset of instability for the periodic structures is defined as the minimum critical strain on all possible $\mathbf{m}=\left(m_{\Theta}, m_{Z}\right)$. Note that the critical strain and the reconstructed mode shapes for each $\mathbf{m}$ shown in the plots are reported on the right.

We find that the critical mode of specimens 1 is associated to $\mathbf{m}=(16 / 8,2)$, resulting in a checkerboard pattern similar to that found in the corresponding full size models and experimental results. Differently, our results indicate that for specimen 2 the critical strain is associated to $\mathbf{m}=(15 / 7,2)$. The corresponding mode is characterized by a pattern similar to that of specimen 1 , but with a line of defects, since the checkerboard pattern is not compatible with an odd number of pores around the circumference. Finally, we find that for specimen 3 the lowest value of critical strain is associated to $\mathbf{m}=$ $(18 / 1,10)$. The critical mode is shown in Fig. 5c-right and is reminiscent of the long wavelength mode observed in the experiments.

In general, the results of Fig. 5 are in excellent agreement with those obtained for the full size models (Fig. 4). Moreover, they indicate that the critical values of strain predicted by the Bloch analysis are slightly larger than those obtained for the full size models. This difference in the prediction of the critical strain is related to both the effect of the boundary conditions at the two ends of the cylinders and the fact that the analysis for the full size models is performed on the unloaded structure, whereas the Bloch wave analysis 

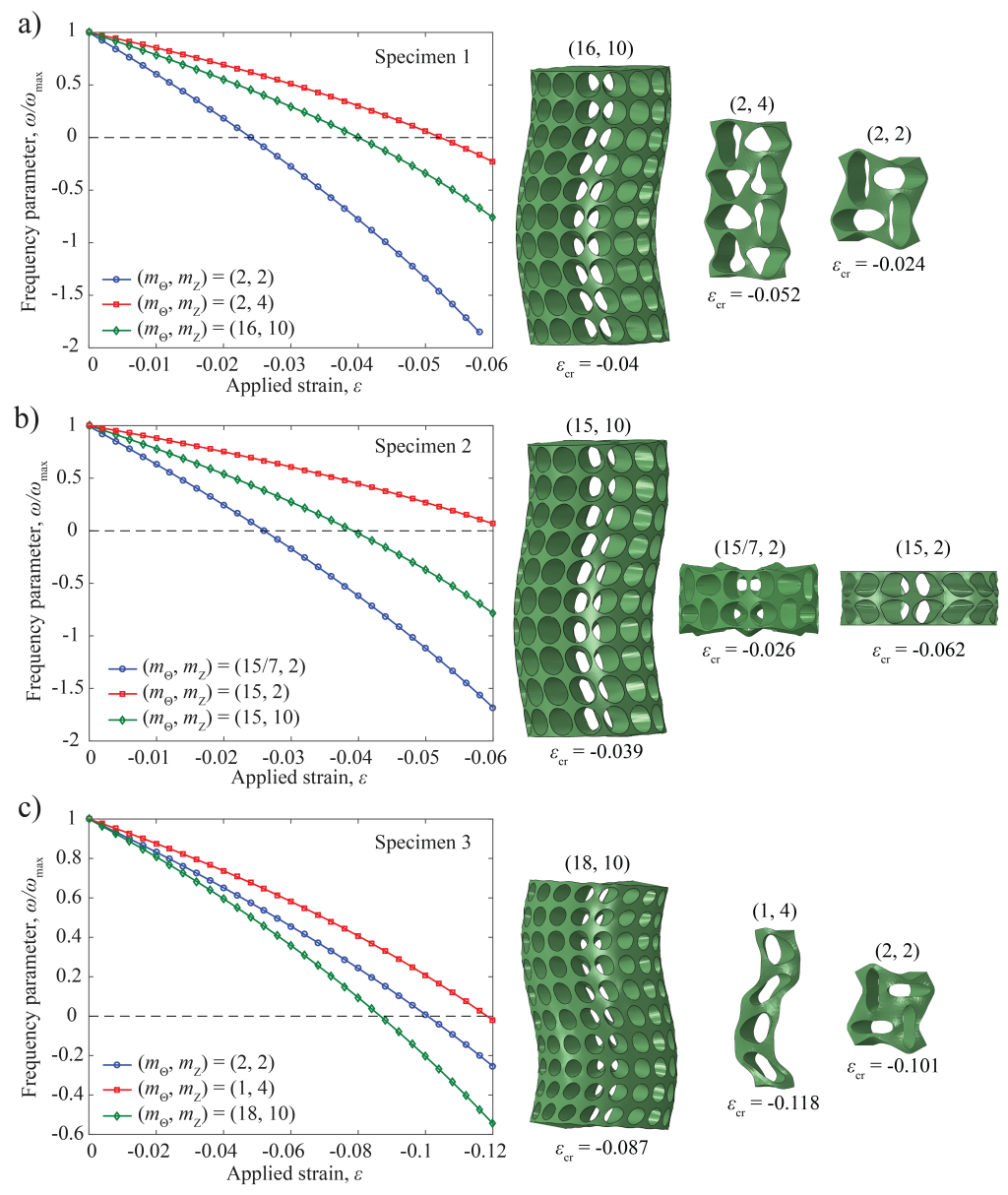

Figure 5: Bloch wave analysis: (a) specimen 1; (b) specimen 2; and (c) specimen 3. On the left, we show the evolution of the frequency parameter as a function of the applied strain for three different values of $\left(m_{\Theta}, m_{Z}\right)$. On the right, we report the mode shapes associated to the considered values of $\left(m_{\Theta}, m_{Z}\right)$. 
accounts for the nonlinear deformation of the structure prior to instability.

\subsection{Post-buckling analysis}

In Fig. 6 the numerical and experimental results of the compression tests for all three specimens are presented, showing excellent agreement. Note that, guided by the results of the Bloch wave analysis, for the unit cell analysis of specimens 1, 2 and 3 we used a supercell comprising an array of $\mathbf{m}=(2,2)$, $\mathbf{m}=(15,2)^{4}$ and $\mathbf{m}=(18,10)$ unit cells, respectively.

All specimens exhibit an initial linear elastic behavior with a sudden departure from linearity to a plateau stress. During the linear elastic range of deformation, the pores undergo a gradual and homogeneous compression. However, as shown by the snapshots on the right side of Fig. 6, for larger values of $\varepsilon$ this relatively affine-like deformation is replaced by a transformation to a pattern of alternating, mutually orthogonal ellipses for specimens 1 and 2. Differently, in specimen 3 buckling deform the entire cylinder into a sinusoidal curve. These transformations correspond to the plateau region immediately after the departure from linearity.

We also note that, when a short wavelength instability is triggered under uniaxial compression as for specimens 1 and 2, the diameter of the structure progressively reduces for increasing values of applied deformation, resulting in an auxetic-like behavior. This unusual behavior can be quantified by inspecting the normalized change of the cylinder outer radius, $\left(r_{\mathrm{o}}-R_{\mathrm{o}}\right) / R_{\mathrm{o}}$, as a function of $\varepsilon$. The results reported in Fig. 7 show that both specimen 1 and 2 significantly contract in radial direction after the onset of instability, while

\footnotetext{
${ }^{4}$ Since, $m_{\Theta}=15 / 7$ is not an integer for specimen 2 , a larger supercell characterized by $\mathbf{m}=(15,2)$ is chosen.
} 
a)
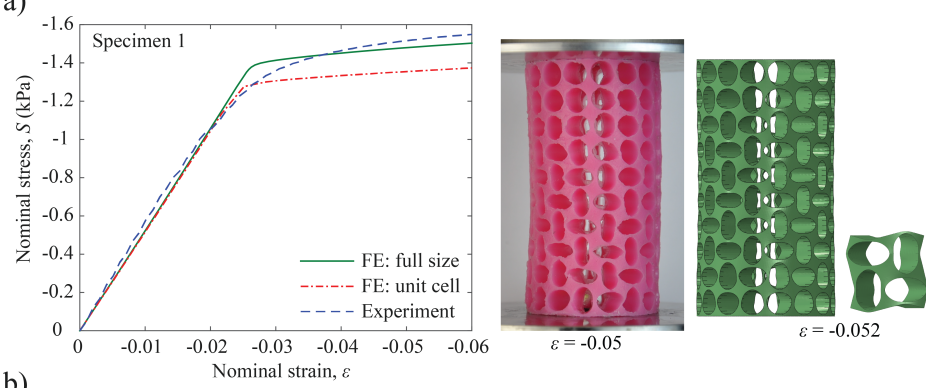

b)
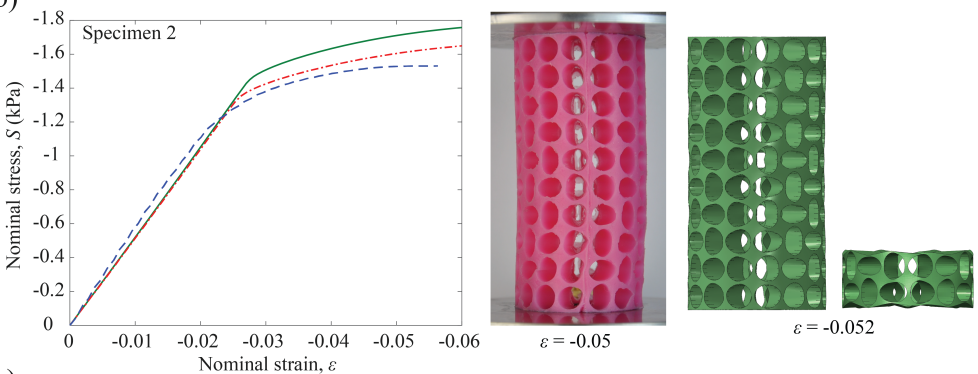

c)
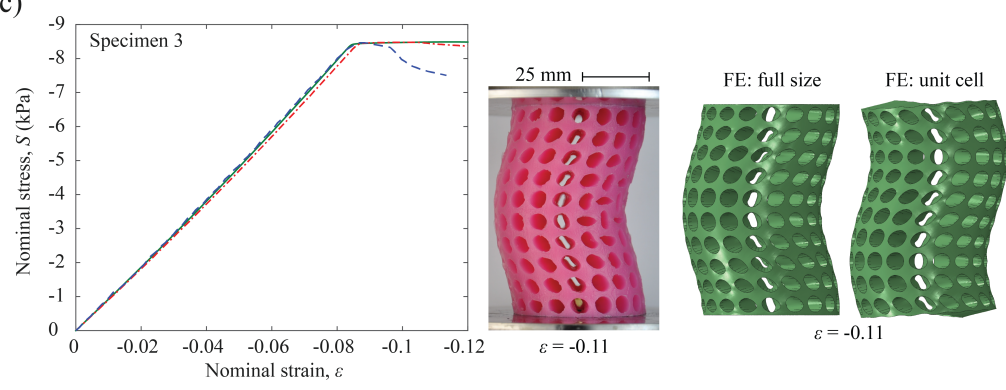

Figure 6: Comparison of numerical and experimental results for uniaxial compression: (a) specimen 1; (b) specimen 2; and (c) specimen 3. On the left, the nominal stress versus nominal strain curves are compared to the experimental data. Snapshots of the samples and the corresponding models are shown on the right. 
specimen 3, which experiences a global buckling, continues to radially expand as the applied strain is increased. It is worth noticing that the evolution of the diameter for specimens 1 and 2 is very similar, indicating that the line of defects introduced because of the odd number of pores in specimen 2 does not significantly alter the macroscopic response of the system. This important observation is also confirmed by the quantitatively similar stress-strain curves shown in Fig. 6a and b for specimen 1 and 2, respectively.

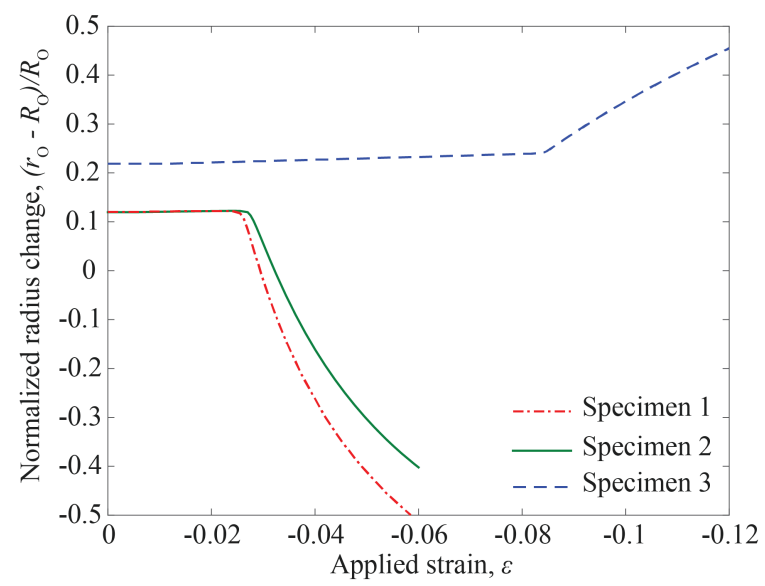

Figure 7: Evolution of the outer radius of the cylinders as a function of the applied compressive deformation. Interestingly, the outer radius of both specimen 1 and specimen 2 starts to decrease above the instability point, resulting in an auxetic-like behavior.

\subsection{Effect of the length and thickness of the porous cylinder}

In this study, we considered three specimens characterized by different values of porosity $(\psi)$ and number of pores around the circumference $\left(N_{\mathrm{c}}\right)$, but with the same inner $\left(R_{i}\right)$ and outer $\left(R_{o}\right)$ radii and rows of pores $\left(N_{r}=\right.$ 10).

To investigate the role played by the length of the cylinder, we calculate the critical strain associated to the short and long wavelength modes for in- 
creasing number of rows. This can be efficiently done by focusing on the unit cell models and calculating the critical strain associated to the modes characterized by $\mathbf{m}=\left(\left\lfloor N_{\mathrm{c}} / 2\right\rfloor, 2\right)$, (short wavelength mode - $\left\lfloor N_{\mathrm{c}} / 2\right\rfloor$ denoting the largest integer smaller than or equal to $\left.N_{\mathrm{c}} / 2\right)$ and $\mathbf{m}=\left(N_{\mathrm{c}}, N_{\mathrm{r}}\right)$ (long wavelength mode) for different values of $N_{r}$. While the local buckling mode is not affected by $N_{r}$, we find that for all three specimens the critical strain associated to the global mode monotonically decreases for increasing values of $N_{r}$ (see Fig. 8). Therefore, long wavelength modes are likely to be triggered in longer cylinders (i.e. for larger values of $N_{r}$ ). More specifically, the results reported in Fig. 8a indicate that in specimen 1 a short wavelength mode is triggered upon compression if $N_{\mathrm{r}}<26$ (as for $N_{\mathrm{r}}<26$ the critical strain associated to the short wavelength mode is lower than that corresponding to the long wavelength mode), while a long wavelength mode is expected for $N_{\mathrm{r}} \geq 26$ (as for $N_{\mathrm{r}} \geq 26$ the critical strain associated to the long wavelength mode is lower than that corresponding to the short wavelength mode). Moreover, we find that the mode switching for specimen 2 and specimen 3 occurs at $N_{\mathrm{r}}=24$ and $N_{\mathrm{r}}=8$, respectively (see Figs. 8b and c). Importantly, our results also indicate that in infinitely long cylinders global modes will always be triggered (as larger values of $m_{Z}$ result in lower critical strains). This observation marks another difference between the response of the porus cylindrical shells considered here and the $2 \mathrm{D}$ counterparts, as a 2D matrix of infinite size perforated with a square array of circular holes can support the local mode for large enough values of porosity (Michel et al., 2007; Bertoldi et al., 2008).

The cylinder thickness, $R_{\mathrm{o}}-R_{\mathrm{i}}$, is another parameter that can signifi- 

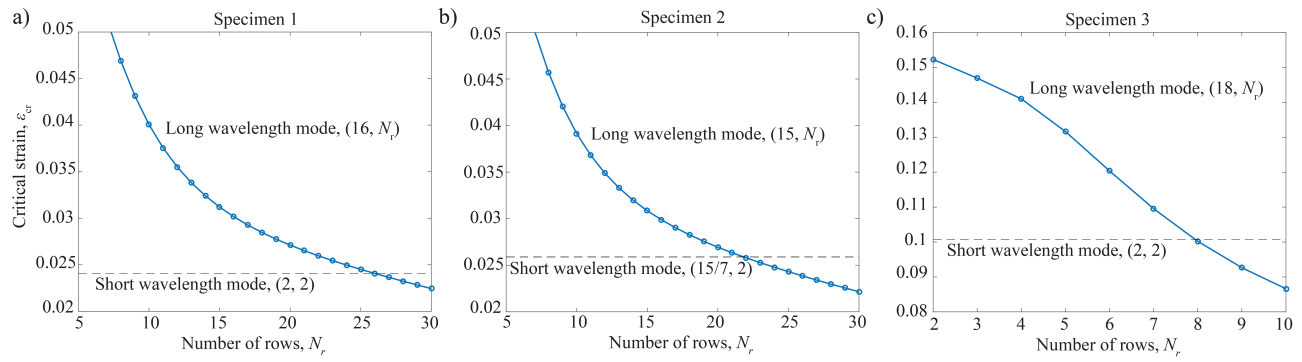

Figure 8: Critical strain associated to the the local and the global modes as a function of $N_{r}$ for : (a) specimen 1; (b) specimen 2; and (c) specimen 3 .

cantly affect the stability of the structure under compression. As shown in Fig. 9, if the thickness of specimen 1 is decreased to $R_{o}-R_{i}=2.5 \mathrm{~mm}$, not only the value of the critical strain is significantly reduced, but also the wavelength of the associated mode is altered. In fact, in this case we find that the critical mode is characterized by $\mathbf{m}=\left(m_{\Theta}, m_{Z}\right)=(8,10)$, resulting in the long wavelength pattern shown in Fig. 9-right.

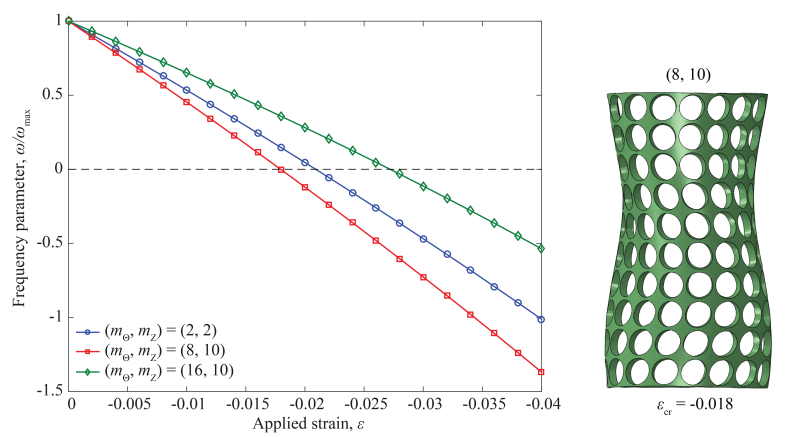

Figure 9: Bloch wave analysis for a cylinder with $N_{\mathrm{c}}=16, \alpha_{p}=0.353, R_{i}=22.5 \mathrm{~mm}$ and $R_{o}=25 \mathrm{~mm}$. On the left, we show the evolution of the frequency parameter as a function of the applied strain for three different values of $\left(m_{\Theta}, m_{Z}\right)$. On the right, we report the mode shape associated to $\left(m_{\Theta}, m_{Z}\right)=(8,10)$. 


\section{Conclusions}

In this work we have combined experiments and numerical analysis to study the non-linear response of cylindrical shells patterned with a square array of circular holes subjected to uniaxial compression. Our results indicate that: $(a)$ the critical buckling mode is highly affected by the geometry of the shell; (b) for long, thin, or low porous cylinders the long wavelength mode (reminiscent of the buckling mode of an Euler-Bernoulli beam) is critical; $(c)$ by decreasing the length and tuning the thickness and porosity of the cylinders a short wavelength instability can be triggered, resulting in a transformation of the initial microstructure into a periodic pattern of mutually orthogonal ellipses; and $(d)$ if the pattern induced by the short wavelength instability is not compatible with the number of pores along the full circumference (as for the case of cylinders with an odd number of pores along the full circumference), a frustrated pattern with a line of defects emerges. The variety of qualitatively different post-buckling behaviors that can be induced in cylindrical patterned shells by controlling their geometry gives us the opportunity to design the next generation of actuators, sensors and switches that take advantage of their highly non-linear response.

\section{Acknowledgments}

This work has been funded by Rolls-Royce Energy. The authors also acknowledge support by the Materials Research Science and Engineering Center under National Science Foundation (NSF) Award No. DMR-1420570 and NSF CMMI-1149456-CAREER award. The authors are also grateful to Huayin $\mathrm{Wu}$ and Professor David Weitz for their support with the rheometer 
experiments. Finally, an anonymous reviewer is thanked for critically reading the manuscript and suggesting substantial improvements.

\section{Appendix A - Uniaxial Tension and Pure Torsion.}

Using the same experimental and numerical setups described in Sections 3 and 4 we also investigate the response of the specimens to uniaxial tension and pure torsion. In particular, to numerically subject the full size models to pure torsion, a cylindrical displacement field $\mathbf{u}=u_{R} \mathbf{e}_{R}+u_{\Theta} \mathbf{e}_{\Theta}+u_{Z} \mathbf{e}_{Z}$ is applied to the nodes on the top surface of the model with components

$$
u_{R}=-R[1-\cos (\gamma L)], \quad u_{\Theta}=R \sin (\gamma L), \quad u_{Z}=0
$$

where $R$ is the initial radial position of each node and $\gamma$ is the applied twist angle per unit length. The corresponding measured torque, $T$, is readily obtained by summing up the reaction forces at all $P$ nodes on the top face as

$$
T=\sum_{i=1}^{P}\left[\xi_{\Theta}^{i} R^{i} \cos (\gamma L)-\xi_{R}^{i} R^{i} \sin (\gamma L)\right],
$$

where $\xi_{\Theta}^{i}$ and $\xi_{R}^{i}$ denote the components of the reaction force at the $i$-th node in circumferential and radial direction, respectively, and $R^{i}$ is the node radial position.

Moreover, for the simulations conducted on unit cell models, pure torsion is simulated by prescribing $\left(\bar{J}_{13}, \bar{J}_{23}, \bar{J}_{33}\right)=(0, \gamma, 0)$. To calculate the

corresponding macroscopic torque, $\bar{T}$, we note that the external work can be expressed as

$$
\delta W_{e x t}=\xi_{2} \delta \bar{J}_{23}=\bar{T} L_{u} \delta \bar{J}_{23}
$$


so that

$$
\bar{T}=\frac{\xi_{2}}{L_{u}}
$$

and $L_{u}$ being defined in Eq. (2).

Results for uniaxial tension. The results for uniaxial tension are presented in Fig. A1 for all three specimens. For each specimen we report the stressstrain curves obtained from the full size (continuous lines) and unit cell (dashdotted lines) analyses and compare them to the experiments (dashed lines). Remarkably, we find an excellent quantitative agreement between all sets of data. All three specimens are characterized by an almost linear stress-strain curve. The curves for specimens 1 and 2 are almost identical, since these structures are characterized by very similar values of porosity $(\psi \sim 0.6)$. In contrast, the curve for specimen 3 is much steeper, indicating that this sample is much stiffer. This is expected, since specimen 3 is characterized by a lower value of porosity $(\psi \sim 0.47)$. Snap-shots of the experiments compared to the full size and unit cell models at the same applied strain, $\varepsilon$, are also shown as insets in each plot. Note that, all specimens show a similar deformation mechanism under tension in which all the pores elongate along the loading direction as the applied deformation progressively increases. The periodicity of the structure, therefore, remains unaltered during loading, so that a single unit cell model is sufficient to accurately capture its response.

Results for pure torsion. FE predictions for pure torsion tests are compared to the experimental results in Fig. A2. In each plot, the normalized torque is shown as a function of the dimensionless applied twist angle per unit length, $R_{o} \gamma$. Note that the torque, $T$, measured during the torsion test is normalized 

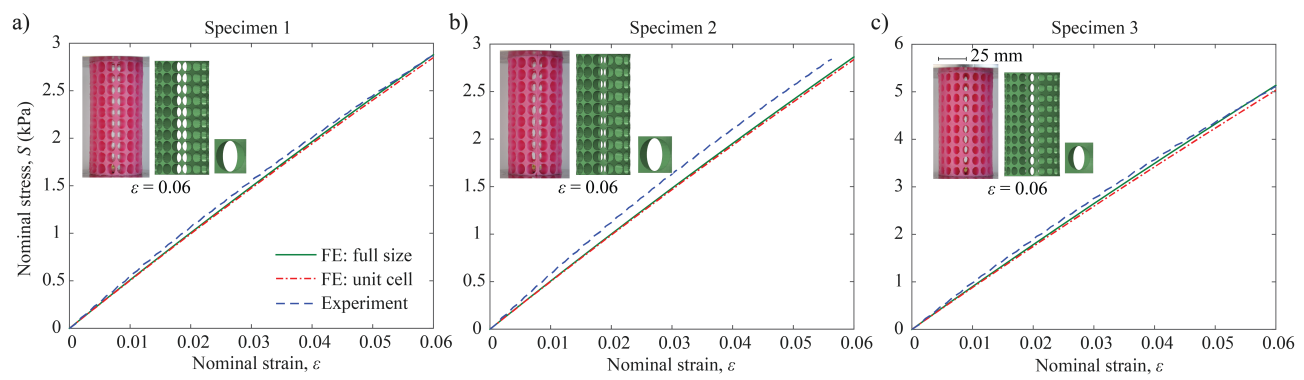

Figure A1: Comparison of numerical and experimental results for uniaxial tension: (a) specimen 1; (b) specimen 2; and (c) specimen 3. The nominal stress versus nominal strain curves, obtained numerically, are compared to the experimental data. Snapshots of the samples and the corresponding models are shown as insets.

as, $T L / J_{c}$, where $J_{c}$ is the polar moment of inertia of the cross-sectional area of the cylinder (i.e. $\left.J_{c}=\pi\left(R_{o}^{4}-R_{i}^{4}\right) / 2\right)$. Again, we find excellent agreement between numerical and experimental results, highlighting the robustness of our numerical approach. Similar to the case of uniaxial tension, we find an almost linear response for all samples. Moreover, specimen 3 is the stiffest specimen also in torsion, given its lower value of porosity. Finally, it should be noted that the maximum values of the normalized twist angles corresponds to 2 radians in specimen 1 and 2 and to 1.75 radian in specimen 3 .
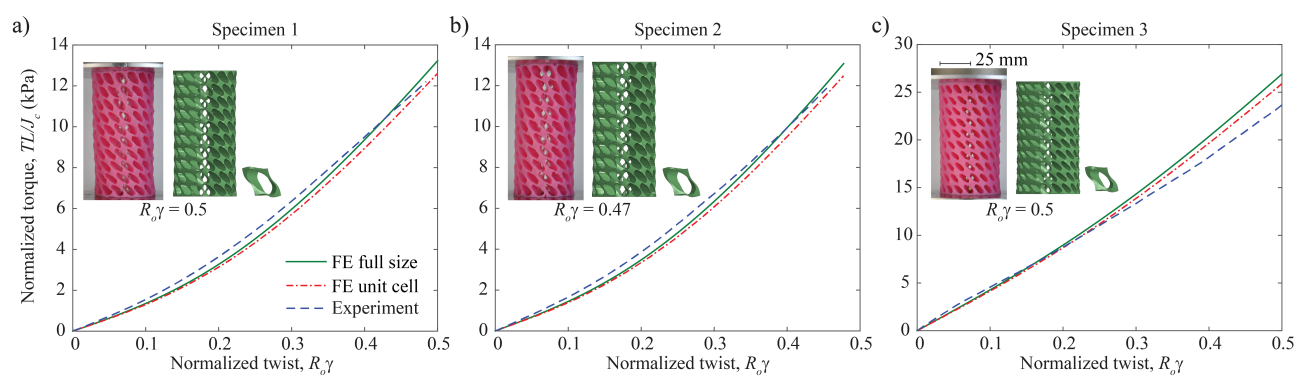

Figure A2: Comparison of numerical and experimental results for pure torsion: (a) specimen 1 ; (b) specimen 2; and (c) specimen 3. Normalized torque versus normalized twist angle. Note that $J_{c}$ is the polar moment of inertia of the cross-sectional area of the cylinder. Snapshots of the samples and the corresponding models are shown as insets. 


\section{Appendix B - Analytical solutions}

Here, we derive analytical expressions for the applied force versus deformation of a homogeneous (non-porous) hollow cylinder subjected to uniaxial loading and pure torsion. We assume incompressibility for our analytical calculations and then compare the resulting expressions to the numerical results obtained from both the full size and the unit cell models. Particularly, we use a nonporous hollow cylinder characterized by $R_{i}=12.5 \mathrm{~mm}, R_{o}=25 \mathrm{~mm}$ and $L=$ $104.72 \mathrm{~mm}$ and also a unit cell with $\gamma=2 \pi / 15$. Moreover, we consider a hyperelastic neo-Hookean material model, as explained in Section 4, for which we can readily found the corresponding Cauchy stress, $\boldsymbol{\sigma}$, as

$$
\boldsymbol{\sigma}=\mathbf{S F}=\mu \mathbf{B}-p \mathbf{I}
$$

where, $\mathbf{S}$ denotes the nominal stress tensor, $\mathbf{F}$ the deformation gradient, $\mathbf{B}=\mathbf{F F}^{T}$ the left Cauchy-Green tensor, $p$ the hydrostatic pressure, and $\mathbf{I}$ the identity matrix.

Uniaxial loading. We begin with the case of axial loading, for which the deformation is uniform throughout the cylinder. If we denote with $\varepsilon$ the applied nominal strain in axial direction, the deformation gradient takes the form

$$
\mathbf{F}=\frac{1}{\sqrt{1+\varepsilon}} \mathbf{e}_{r} \otimes \mathbf{e}_{R}+\frac{1}{\sqrt{1+\varepsilon}} \mathbf{e}_{\theta} \otimes \mathbf{e}_{\Theta}+(1+\varepsilon) \mathbf{e}_{z} \otimes \mathbf{e}_{Z}
$$


Substituting Eq. (B2) into Eq. (B1) and imposing $\sigma_{r r}=\sigma_{\theta \theta}=0$, the Cauchy stress is found as

$$
\boldsymbol{\sigma}=\mu\left[(1+\varepsilon)^{2}-\frac{1}{1+\varepsilon}\right] \mathbf{e}_{z} \otimes \mathbf{e}_{z}
$$

In Fig. B1 we compare FE results obtained from the full size and unit cell models with the analytical predictions. Note that the small discrepancy found for the FE results of the full size model is dictated by the boundary conditions used in those analysis, where the expansion in radial direction at the two ends of the cylinder is completely prevented.

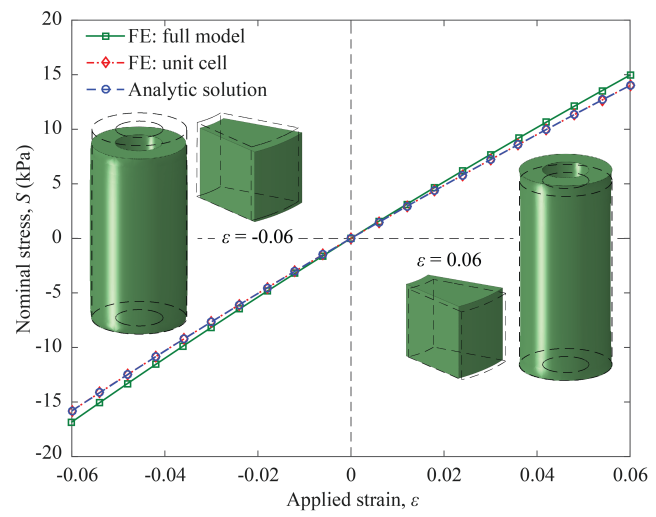

Figure B1: Homogeneous (non-porous) cylinder under uniaxial loading: comparison of the analytical solution with numerical results obtained from the full size and unit cell models.

Pure torsion. Under pure torsion, the deformation in cylindrical coordinates takes the form

$$
r=r(R), \quad \theta=\Theta+\gamma Z, \quad z=Z,
$$


where $\gamma$ is the twist per unit length of the deformed cylinder and $r(R)$ is a function of the original radial coordinate $R$. It follows that the deformation gradient in cylindrical coordinates is given by

$$
\mathbf{F}=\frac{\mathrm{d} r}{\mathrm{~d} R} \mathbf{e}_{r} \otimes \mathbf{e}_{R}+\frac{r}{R} \mathbf{e}_{\theta} \otimes \mathbf{e}_{\Theta}+\gamma r \mathbf{e}_{\theta} \otimes \mathbf{e}_{Z}+\mathbf{e}_{z} \otimes \mathbf{e}_{Z}
$$

Moreover, since the material is incompressible, we will have

$$
r^{2}-r_{i}^{2}=R^{2}-R_{i}^{2}
$$

where $r_{i}$ and $R_{i}$ denote the inner radius of the cylinder in the current and reference configurations, respectively. Note that Eq. (B6) can be rewritten in differential form as

$$
\frac{\mathrm{d} r}{\mathrm{~d} R}=\frac{R}{r}
$$

Substituting Eqs. (B5) and (B7) into Eq. (B1) the stress components can be obtained as

$$
\begin{aligned}
\sigma_{r \theta}=\sigma_{r z} & =0, \quad \sigma_{\theta z}=\mu \gamma r, \quad \sigma_{r r}=\mu \frac{R^{2}}{r^{2}}-p \\
\sigma_{\theta \theta} & =\mu\left(\frac{r^{2}}{R^{2}}+\gamma^{2} r^{2}\right)-p, \quad \sigma_{z z}=\mu-p,
\end{aligned}
$$

where the pressure $p$ here is a function of $r$. Moreover, since equilibrium requires that

$$
\frac{\mathrm{d} \sigma_{r r}}{\mathrm{~d} r}+\frac{\sigma_{r r}-\sigma_{\theta \theta}}{r}=0,
$$


by substituting Eqs. (B8) into Eqn. (B9) we obtain

$$
\frac{\mathrm{d} p}{\mathrm{~d} r}=\mu\left(-\frac{R^{2}}{r^{3}}+\frac{2}{r}-\frac{r}{R^{2}}-\gamma^{2} r\right)
$$

Finally, $p$ can be obtained by integrating Eq. (B10) and using Eq. (B7),

$$
p=\mu\left(\frac{1}{2} \frac{R^{2}}{r^{2}}+\ln \frac{r}{R}-\frac{1}{2} \gamma^{2} r^{2}+C_{p}\right)
$$

where $C_{p}$ is a constant. The two constants $C_{p}$ (in Eq. (B11)) and $r_{i}$ (in Eq. (B6)), can be then determined by imposing that the pressure on the inner and outer surfaces of the cylinder is zero

$$
\sigma_{r r}\left(r=r_{i}\right)=\sigma_{r r}\left(r=r_{o}\right)=0
$$

Substituting Eqns. (B8), (B11) and (B6) into Eq. (B12), we obtain a nonlinear system of equations from which we can solve for $C_{p}$ and $r_{i}$,

$$
\left\{\begin{array}{c}
\frac{1}{2} \frac{R_{i}^{2}}{r_{i}^{2}}-\ln \frac{r_{i}}{R_{i}}+\frac{1}{2} \gamma^{2} r_{i}^{2}-C_{p}=0, \\
\frac{1}{2} \frac{R_{o}^{2}}{r_{i}^{2}+R_{o}^{2}-R_{i}^{2}}-\ln \frac{\sqrt{r_{i}^{2}+R_{o}^{2}-R_{i}^{2}}}{R_{o}}+\frac{1}{2} \gamma^{2}\left(r_{i}^{2}+R_{o}^{2}-R_{i}^{2}\right)-C_{p}=0 .
\end{array}\right.
$$

Finally, the torque exerted on the cylinder can be readily obtained as

$$
M=\int_{r_{i}}^{\sqrt{r_{i}^{2}+R_{o}^{2}-R_{i}^{2}}} 2 \pi r \sigma_{\theta z} r \mathrm{~d} r=\frac{\pi}{2} \mu \gamma\left[\left(r_{i}^{2}+R_{o}^{2}-R_{i}^{2}\right)^{2}-r_{i}^{4}\right]
$$

Numerical and analytical results are compared in Fig. B2, showing excellent agreement and confirming the validity of the boundary conditions used in our simulations. 


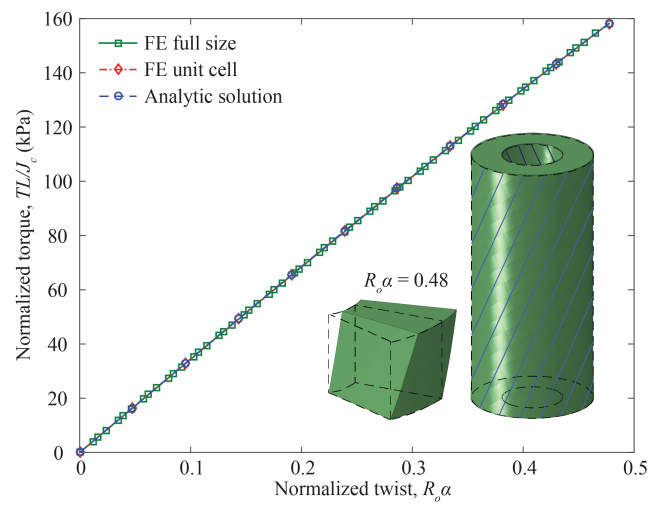

Figure B2: Homogeneous (non-porous) cylinder under pure torsion: comparison of the analytical solution with numerical results obtained from the full size and unit cell models.

\section{References}

Aberg, M., Gudmundson, P., 1997. The usage of standard finite element codes for computation of dispersion relations in materials with periodic microstructure. Journal of the Acoustical Society of America 102 (4), 20072013.

Bertoldi, K., Boyce, M. C., Deschanel, S., Prange, S. M., Mullin, T., 2008. Mechanics of deformation-triggered pattern transformations and superelastic behavior in periodic elastomeric structures. Journal of the Mechanics and Physics of Solids 56, 2642-2668.

Bertoldi, K., Reis, P. M., Willshaw, S., Mullin, T., 2010. Negative poisson's ratio behavior induced by an elastic instability. Advanced Materials 22 (3), $361-366$.

Gibson, L., Ashby, M., 1999. Cellular Solids: Structure and Properties. Cambridge University Press. 
Kang, S. H., Shan, S., Noorduin, W. L., Khan, M., Aizenberg, J., Bertoldi, K., 2013. Buckling-induced reversible symmetry breaking and amplification of chirality using supported cellular structures. Advanced Materials 25 (24), 3380-3385.

Kittel, C., 2005. Introduction to solid state physics, 8th Edition. Wiley, Hoboken, NJ.

Lazarus, A., Reis, P. M., 2015. Soft actuation of structured cylinders through auxetic behavior. Advanced Engineering Materials 17 (6), 815-820.

Maldovan, M., Thomas, E. L., 2009. Periodic materials and interference lithography: for photonics, phononics and mechanics. John Wiley \& Sons.

Michel, J. C., Lopez-Pamies, O., Castañeda, P. P., Triantafyllidis, N., 2007. Microscopic and macroscopic instabilities in finitely strained porous elastomers. Journal of the Mechanics and Physics of Solids 55 (5), 900-938.

Mullin, T., Deschanel, S., Bertoldi, K., Boyce, M., 2007. Pattern transformation triggered by deformation. Physical Review Letters 99, 084301.

Ning, X., Pellegrino, S., 2015. Buckling analysis of axially loaded corrugated cylindrical shells. In: 56th AIAA/ASCE/AHS/ASC Structures, Structural Dynamics, and Materials Conference. p. 1435.

Papka, S. D., Kyriakides, S., 1994. In-plane compressive response and crushing of honeycomb. Journal of the Mechanics and Physics of Solids 42 (10), 1499-1532. 
Prasad, M., Pattabiraman, J., Rao, B., 1974. A note on dipole idealization of transversely vibrating beams. Journal of Sound and Vibration 37 (2), 280-283.

Queheillalt, D. T., Wadley, H. N., 2005. Cellular metal lattices with hollow trusses. Acta Materialia 53 (2), 303-313.

Shim, J., Perdigou, C., Chen, E. R., Bertoldi, K., Reis, P. M., 2012. Bucklinginduced encapsulation of structured elastic shells under pressure. Proceedings of the National Academy of Sciences of the United States of America 109 (16), 5978-5983.

Thomas, D. L., 1979. Dynamics of rotationally periodic structures. International Journal for Numerical Methods in Engineering 14 (1), 81-102.

Triantafyllidis, N., Nestorovic, M., Schraad, M., 2006. Failure surfaces for finitely strained two-phase periodic solids under general in-plane loading. Journal of Applied Mechanics 73, 505-515.

Verdejo, R., Stämpfli, R., Alvarez-Lainez, M., Mourad, S., Rodriguez-Perez, M., Brühwiler, P., Shaffer, M., 2009. Enhanced acoustic damping in flexible polyurethane foams filled with carbon nanotubes. Composites Science and Technology 69 (10), 1564-1569.

Wierzbicki, T., Abramowicz, N., 1983. On the crushing mechanics of thinwalled structures. Journal of Applied Mechanics 50, 727-734.

Williams, F., Anderson, M., 1983. Incorporation of lagrangian multipliers into an algorithm for finding exact natural frequencies or critical buckling loads. International Journal of Mechanical Sciences 25 (8), 579-584. 
Zhang, Y., Matsumoto, E. A., Peter, A., Lin, P., Kamien, R. D., Yang, S., 2008. One-step nanoscale assembly of complex structures via harnessing of an elastic instability. Nano Letters 8 (4), 1192-1196.

Zhu, X., 2011. Design and fabrication of photonic microstructures by holographic lithography and pattern transformation. Ph.D. thesis, University of Pennsylvania. 